\title{
Clinical Study about Latissimus Dorsi Based Breast Reconstruction at a Tertiary Health Centre in Mangalore, India
}

\author{
Gopinathan S. ${ }^{1}$ \\ ${ }^{1}$ Department of Surgical Oncology, Father Muller Medical College, Mangalore, Karnataka, India.
}

\section{ABSTRACT}

\section{BACKGROUND}

The most common cancer among urban Indian women is breast cancer. Mastectomy causes loss of body image and negatively impacts the quality of life. Restoration of body image is an important step for breast cancer survivors. Recent reconstruction options are autologous or implant-based reconstruction or combined approach. This present clinical study was done to describe about latissimus dorsi based breast reconstruction (BR) at a tertiary health centre.

\section{METHODS}

This retrospective study was conducted at a tertiary health care centre from Jun 2018 to Dec 2020. Eligible desiring patients' divided into the small, medium and large breasts and were willing to undergo BR were taken up for surgery. Patients considered as suitable candidates were further assessed for the option of reconstruction by latissimus dorsi (LD) flap, LD flap with an implant. All the patients were fully explained about the procedure.

\section{RESULTS}

The total number of mastectomies were 158 and breast reconstruction was done in 45 patients, with a percentage of $29 \%$. In our study, majority of the patients $(91.1$ $\%)$ underwent immediate breast reconstruction. Reconstructive methods used in our study were autologous LD flap (64.4\%), LD flap with silicone implant (24.2\%) and pedicled transverse rectus abdominis myocutaneous (TRAM) flap (11.2\%) based on the size of the contralateral breast. The most common type of reconstruction in our study was the LD flap (64.4\%).

\section{CONCLUSIONS}

Immediate reconstruction of mastectomy defects was offered to all interested patients. Autologous or implant-based can be done safely with minimum morbidity. Latissimus dorsi flap-based breast reconstruction is a cost-effective, safe, reliable method at a tertiary health centre.

\section{KEY WORDS}

Breast Reconstruction, Flap, LD Flap, Implant
Corresponding Author: Dr. Gopinathan $S$., Department of Surgical Oncology, Father Muller Medical College, Mangalore, Karnataka, India. E-mail: dr.s.gopinathan@gmail.com

DOI: $10.14260 / j e m d s / 2021 / 592$

How to Cite This Article:

Gopinathan S. Clinical study about latissimus dorsi based breast reconstruction at a tertiary health centre in Mangalore, India. J Evolution Med Dent Sci 2021;10(34):2905-2909, DOI: 10.14260/jemds/2021/592

Submission 25-03-2021,

Peer Review 29-05-2021, Acceptance 05-06-2021, Published 23-08-2021.

Copyright (c) 2021 Gopinathan S. This is an open access article distributed under Creative Commons Attribution License [Attribution 4.0 International (CC BY 4.0)] 


\section{BACKGROUND}

The most common cancer among urban women in India is breast cancer. ${ }^{1}$ The gold standard treatment of operable breast cancer is breast conservation surgery (BCS) or modified radical mastectomy with or without reconstruction. Mastectomy causes loss of body image and negatively impacts quality of life. For the rehabilitation of these patients, restoration of body image is an important step..$^{2,3}$ Breast reconstruction (BR) is done in a small percentage of patients due to cost, surgical training, and technical skill.

Recently, various options of BR are available. The options are autologous or implant-based reconstruction or combined approach. Autologous BR choices are TRAM, LD flap, DIEP (Deep Inferior epigastric) flap or Superior epigastric flap. Implant based reconstruction choices are silicone-filled or saline-filled expanders. ${ }^{4}$ Each of these reconstructive methods has its benefits and drawbacks. The ideal breast reconstruction following a mastectomy should be a one-stage procedure; restore deficient skin; replace the contour of the absent pectoralis major muscle; restore the lost breast volume; replace the absent nipple-areolar complex, and match symmetrically and pleasingly the contour of the remaining breast.

In 1897 Iginio and Tansini first developed a workhorse flap that is LD flap to reconstruct the post-mastectomy defects. ${ }^{5}$ Since then, there have been variety of advancements in the harvesting and inserting technique of LD Flap. McCraw and Pepp and delay and colleagues' modifications gave extra bulk of flap which was suitable for medium breast reconstruction without using an implant. ${ }^{6}$

Silicone breast implants were introduced in the 1960 s and offered the first opportunity to provide a reconstruction of the breast following a mastectomy. During those times, extirpative surgery resulted in significant breast deformities due to the more radical approaches used, and the breast implants allowed for really nothing more than the recreation of a breast mound. In the late 1970s, the LD flap was reintroduced and combined with implant placement to provide notably improved results in breast reconstruction. ${ }^{7,8}$ Rather than simply placing an implant beneath thin skin flaps, the latissimus muscle served to replace or even augment the pectoralis muscle to provide coverage of the implant.9,10

This present clinical study was done to describe about latissimus dorsi based breast reconstruction (BR) at a tertiary health centre.

\section{METHODS}

This retrospective study was conducted at a tertiary health care centre from Jun 2018 to Dec 2020. Patients work up were history, clinical examination, and metastatic workup. Patients considered as suitable candidates were further assessed for the option of reconstruction by LD flap, LD flap with an implant. Eligible desiring patients' were divided into the small, medium and large breasts (Based on subjective assessment and bulk of reconstruction required) and were willing to undergo BR were taken up for surgery. All the patients were fully explained about the procedure.
Small breast patients were offered LD flap, medium and large breast patients were offered LD flap + Implant. First, patients underwent MRM and special attention was paid to maintain the integrity of the thoracodorsal pedicle during the axillary dissection. Care was also taken not to disturb the inframammary fold as well the fascial attachment along the lateral chest wall.

For LD flap harvesting, patients were turned to lateral decubitus position with $90^{\circ}$ abducted shoulder. The incisions on the donor site were made down to the subdermal layer. The plane of dissection was kept along the subcutaneous plane just above Scarpa's fascia, maintaining a thickness of skin flap of at least $1 \mathrm{~cm}$. Fat was harvested from the scapular and the iliac region as much as possible, to lift the largest possible flap in terms of volume. Then the flap was brought to defect through the axilla.

Implant size was measured based on the size of the contralateral breast size assessed and kept ready before mastectomy. Then LD harvesting was done from the abovementioned method and placed above the implant. The flap was covering the implant all around. Immediate and delayed seroma aspiration, wound resuturing were recorded and complications related to recipients' site and donor site were assessed.

Cosmesis was assessed by two surgeons and recorded as excellent, good and fair. Patients expressed cosmesis assessment as very satisfied, satisfied and fair (patient's satisfaction). Data was compiled in MS Excel and checked for completeness and correctness. Then it was analysed.

Statistical analysis was done by the descriptive and casual analysis method.

\section{RESULTS}

Total number of mastectomies were 158 and breast reconstruction was done in 45 patients, with a percentage of $29 \%$. The most common age group was less than 40 years $(48.9 \%)$ and the majority was the premenopausal group (Table 1). The right breast was commonly presented with carcinoma and increased incidence of T4, N1 stage with a predominance of infiltrative ductal carcinoma (Table 2). In our study, surgical procedures done were Pates mastectomy (62.2\%), skin-sparing mastectomy (26.7\%), nipple-sparing mastectomy (6.7 \%) and radical mastectomy (4.4\%). Radical or modified radical mastectomy was done by transverse or oblique incision whereas skin-sparing or nipple sparing mastectomy was done by Tennis bat-shaped incision (Table 3). Majority $(91.1 \%)$ of the patients underwent immediate breast reconstruction. Reconstructive methods used in our study were autologous LD flap (71.1\%), LD flap with silicone implant (28.9\%) based on size of the contra lateral breast (Table 4). Most common type of reconstruction in our study was LD flap (71.1\%).

Most common procedure related complication observed at donor and recipient sites was seroma followed by wound dehiscence. 5 patients developed partial flap loss and no patient had total flap loss (Table 5). Cosmetic status assessed by patients was rated as very satisfied, satisfied, and fair and a majority of patients were satisfied (66.7\%). Surgeon's 
score assessed as excellent (15.6\%), good (62.2\%), and fair (22.2\%) (Table 6).

\begin{tabular}{|ccc|}
\hline Age & No & Percentage (\%) \\
\hline < 40 Yrs. & 22 & $48.9 \%$ \\
$40-50$ Yrs. & 18 & $40.0 \%$ \\
> 50 Yrs. & 05 & $11.1 \%$ \\
\hline \multicolumn{3}{|c}{} \\
\hline
\end{tabular}

\begin{tabular}{|ccc|}
\hline Menstrual Status & No & Percentage \\
Pre-menopausal & 40 & $88.9 \%$ \\
Post-menopausal & 05 & $11.1 \%$ \\
& Table 2. Tumour Characteristics & \\
Side & No & $\%$ \\
Right & 28 & $62.2 \%$ \\
Left & 17 & $37.8 \%$ \\
T Stage & Table 2(a). Side & \\
T1 & No & $\%$ \\
T2 & 03 & $06.7 \%$ \\
T3 & 05 & $11.1 \%$ \\
T4 & 15 & $33.3 \%$ \\
N Stage & 22 & $48.9 \%$ \\
N1 & No & $\%$ \\
N2 & 31 & 68.9 \\
N3 & 12 & 26.7 \\
Types & 02 & 04.4 \\
IDC & Table 2(b). Stage & $\%$ \\
ILC & No & 82.2 \\
COLLOID & 37 & 06.7 \\
& 03 & 06.7 \\
\hline & 03 & \\
\hline
\end{tabular}

\begin{tabular}{|ccc|}
\hline Incisions & No & $\%$ \\
Transverse & 25 & 55.6 \\
Oblique & 09 & 20.0 \\
& Tennis Shaped & \\
Table 3. Primary Surgery & Characteristics (a). Type of Incision for Mastectomy \\
Types & No & $\%$ \\
Patey's mastectomy & 28 & 62.2 \\
Skin sparing mastectomy & 12 & 26.7 \\
Nipple sparing mastectomy & 03 & 06.7 \\
Radical mastectomy & 02 & 04.4 \\
\hline \multicolumn{2}{|c}{ Table 3 (b). Type of Mastectomy } \\
\hline
\end{tabular}

\begin{tabular}{|c|c|c|c|c|c|}
\hline \multicolumn{2}{|l|}{ Timing } & \multicolumn{2}{|r|}{ No } & \multicolumn{2}{|r|}{$\%$} \\
\hline \multicolumn{2}{|l|}{ Immediate } & \multicolumn{2}{|r|}{41} & \multicolumn{2}{|r|}{91.1} \\
\hline \multicolumn{2}{|l|}{ Delayed } & \multicolumn{2}{|r|}{04} & \multicolumn{2}{|r|}{08.9} \\
\hline \multicolumn{6}{|c|}{ Table 4. Reconstruction Characteristics, (a). Timing of Breast Reconstruction } \\
\hline Types & No & $\%$ & Small Breast & Medium Breast & t Large Breast \\
\hline LD Flap & 32 & 71.1 & 20 & 12 & 00 \\
\hline LD FLAP+ Implant & 13 & 28.9 & 00 & 10 & 02 \\
\hline \multicolumn{6}{|c|}{ Table 4 (b). Type of Reconstruction } \\
\hline
\end{tabular}

\begin{tabular}{|ccc|}
\hline Complications & LD Flap & LD flap+ Implant \\
Hematoma & 01 & 01 \\
Wound Infection & 02 & 00 \\
Fat necrosis & 01 & 00 \\
Reopen & 01 & 01 \\
Total flap loss & 00 & 00 \\
Partial flap loss & 03 & 01 \\
Seroma & 08 & 04 \\
Wound dehiscence & 04 & 00 \\
Table 5. Procedure Related Complications (a). Early Complications \\
Complications & LD Flap & LD flap+ Implant \\
Hypertrophic scar & 01 & 02 \\
Malposition & 00 & 00 \\
Capsular contracture & 00 & 00 \\
\multicolumn{3}{c}{ Table 5(b). Late Complications } \\
Procedures & LD Flap & LD flap+ Implant \\
Scar revision & 02 & 01 \\
Redo flap & 00 & 00 \\
\hline \multicolumn{2}{c}{ Table 5 (c). Secondary Procedure } \\
\hline \multicolumn{2}{c}{} \\
\hline
\end{tabular}

\begin{tabular}{|ccc|}
\hline Chemotherapy & No & $\%$ \\
Received & 25 & 55.6 \\
Not received & 20 & 44.4 \\
Table 6. Chemotherapy Status, (a). NACT Status & \\
Chemotherapy & No & $\%$ \\
Received & 38 & 84.4 \\
Not received & 07 & 15.6 \\
\hline Table 6. Chemotherapy Status (b). Adjuvant Chemo \\
\hline
\end{tabular}

\section{DISCUSSION}

The main aim of breast reconstruction is to create a soft, symmetric, sensible, aesthetically acceptable breast with minimum donor morbidity. The optimal method of BR should be soft, reliable and with no health risk. The breast reconstruction rate in India is lower compared to the west (80-90\%). It is mainly due to patient education, cost, and surgeons training. The reconstruction rate in our study was $29 \%$ only. Immediate reconstruction advantages are better aesthetic result, psychological effect, lower cost and disadvantages are high risk of complications. Delayed reconstruction advantages are lower complication rate and disadvantages are less optimal result, high percentage of anxiety, depression and impairment of their sexual attractiveness and higher cost (2 operations, hospital stays). ${ }^{11}$

LD flap is one of the most reliable and versatile methods of BR. The aesthetic results from bulky LD reconstructions are superior to reconstruction with implants due to their more natural appearance, consistency, and durability. Autologous tissue can also withstand RT better. Functional impairment after LD flap harvest is also minimal and affects only very specific activities like rowing, cross country skiing or mountain climbing, but appears to have little effect on most other activities. One of the limitations of autologous LD flap is the volume of the harvested tissue and it is usually not possible to match the size of a large breast. Some workers have reported on the harvesting of superficial fibres of serratus anterior muscle along with overlying fat to increase the volume of the flap. ${ }^{12}$ Lip modelling by Coleman fat transfer can also be another method to augment the flap volume. ${ }^{13}$ However, an ideal patient for autologous LD reconstruction should have a small or medium size breast.

LD flap with implant - Among the currently available options, implant-based reconstruction is most commonly performed. However, they are expensive and have their aesthetic limitations. Implant-based reconstructions are usually round and natural ptosis is difficult to reproduce. Therefore, in approximately $60 \%$ of cases, it is necessary to perform a contralateral mammoplasty to improve the symmetry. ${ }^{14}$ Another major drawback of implant-based reconstruction is capsular contracture, which occurs in at least $15 \%$ of patients after a follow up of 2 years. ${ }^{9}$ In severe cases of capsular contracture, it may even be necessary to perform a surgical capsulotomy. ${ }^{15}$

Complications associated with LD flap; Implants are manageable. Donor site morbidity is another potential problem of this flap. Seroma collection and skin necrosis of the dorsal skin flaps have been variably reported by several authors. ${ }^{16,17,18}$ Seroma formation is common after the harvest of the autologous LD flap. Several strategies have been suggested to reduce the incidence of post-operative seroma such as quilting sutures and injection of topical fibrin glue. ${ }^{19,20}$ In our series, four patients (21\%) had breakdown of suture line and two (10.5\%) had partial necrosis of skin flaps. Delay et al. reported $3 \%$ incidence in 100 patients, ${ }^{16}$ while Chang et al. reported $16 \%$ necrosis rate in 75 patients. ${ }^{17}$ To avoid this complication, it is important that primary wound closure of the donor site should be relatively 
tense free and the width of the skin paddle should not exceed $6 \mathrm{~cm}$.

In our study, 5 patients developed partial flap loss at the edges and were easily managed with debridement. As per literature, chemotherapy and radiotherapy increase the flap related complications, but in our study, majority received chemotherapy and radiotherapy and complications were minimal and manageable. Post-mastectomy radiation, irrespective of the method of reconstruction, increases the incidence of postoperative complications; however, this study demonstrates that an autologous flap offers a more favourable outcome in terms of morbidity than expander. ${ }^{21}$

Valid tools to assess aesthetic outcomes after breast reconstructive surgery are scarce. Several studies report aesthetic outcomes, in the form of an assessment by the patient, by the surgeon, or by an independent professional. ${ }^{22-}$ 26 Some authors use questionnaires, whereas others use photographs to assess aesthetic outcomes. However, measures for the assessment of aesthetic outcomes of breast reconstruction vary widely between studies and are often illdefined. We used assessment by a patient and two surgeons. Majority of the patients were satisfied. Reconstruction options can be given to economically poor patients as modified LD flap.

\section{CONCLUSIONS}

Immediate reconstruction of mastectomy defects can be offered to all interested patients. Autologous or implantbased reconstruction can be done safely with minimum morbidity. Latissimus dorsi flap-based breast reconstruction is a cost-effective, safe method and reliable in a tertiary health centre.

Data sharing statement provided by the authors is available with the full text of this article at jemds.com.

Financial or other competing interests: None.

Disclosure forms provided by the authors are available with the full text of this article at jemds.com.

\section{REFERENCES}

[1] Raina V, Bhutani M, Bedi R, et al. Clinical features and prognostic factors of early breast cancer at amajor centre in North India. Indian J Cancer 2005;42(1):40-5.

[2] Elder EE, Brandberg Y, Bjorklund T, et al. Quality of life and patient satisfaction in breast cancer patients after immediate breast reconstruction: a prospective study. Breast 2005;14(3):201-8.

[3] Dian D, Schwenn K, Mylonas I, et al. Quality of lifeamong breast cancer patients undergoing autologous breast reconstruction versus breast conserving therapy. J Cancer Res Clin Oncol 2007;133(4):247-52.

[4] Petit JY, Rietjens M, Lohsiriwat V, et al. Update on breast reconstruction techniques and indications. World J Surg 2012;36(7):1486-97.

[5] Maxwell GP. Iginio Tansini and the origin of the latissimus dorsi musculocutaneous flap. Plast Reconstr Surg 1980;65(5):686-92.
[6] McCraw JB, Pepp CT. Latissimus dorsi myo-cutaneous flap: 'Fleur de lys' reconstruction. In: Hartrampf CR, edr. Breast reconstruction with living tissues. Norfolk, VA: Hamptom Press 1991: p. 211.

[7] Bostwick J 3rd, Vasconez LO, Jurkiewicz MJ. Breast reconstruction after a radical mastectomy. Plast Reconstr Surg 1978;61(5):682-93.

[8] Radovan C. Breast reconstruction after mastectomy using the temporary expander. Plast Reconstr Surg 1982;69(2):195-208.

[9] De la Torre JI, Fix RJ, Gardner PM, et al. Reconstruction with the latissimus dorsi flap after skin-sparing mastectomy. Ann Plast Surg 2001;46(3):229-33.

[10] Parikh PM, Spear SL, Menon N, et al. 20: immediate breast reconstruction with tissue expanders and alloderm. Plast Reconstr Surg 2006;118(Suppl 4):18.

[11] Chevray PM. Timing of breast reconstruction: immediate vs. delayed. Cancer J 2008;14(4):223-9.

[12] Denewer A, Setit A, Hussein 0, et al. Skin-sparing mastectomy with immediate breast reconstruction by a new modification of extended latissimus dorsi myocutaneous flap. World J Surg 2008;32(12):2586-92.

[13] Delay E. Lipomodelling of the reconstructed breast. In: Spear SL, edr. Surgery of the breast: principles and art. $2^{\text {nd }}$ edn. Philadelphia, PA: Lippincott-Raven 2006: p. 930.

[14] Petit JY, Rietjens M, Lohsiriwat V, et al. Update on breast reconstruction techniques and indications. World J Surg 2012;36(7):1486-97.

[15] Nguyen JT, Carey JN, Wong AK. Use of human acellular dermal matrix in implant-based breast reconstruction: evaluating the evidence. J Plast Reconstr Aesthet Surg 2011;64(12):1553-61.

[16] Delay E, Gounot N, Bouillot A, et al. Autologous latissimus breast reconstruction: a 3-year clinical experience with 100 patients. Plast Reconstr Surg 1998;102(5):1461-78.

[17] Chang DW, Youssef A, Cha S, et al. Autologous breast reconstruction with the extended latissimus dorsi flap. Plast Reconstr Surg 2002;110(3):751-9; discussion 7611.

[18] Clough KB, Louis-Sylvestre C, Fitoussi A, et al. Donor site sequelae after autologous breast reconstruction with an extended latissimus dorsi flap. Plast Reconstr Surg 2002;109(6):1904-11.

[19] Dancey AL, Cheema M, Thomas SS. A prospective randomized trial of the efficacy of marginal quilting sutures and fibrin sealant in reducing the incidence of seromas in the extended latissimus dorsi donor site. Plast Reconstr Surg 2010;125(5):1309-17.

[20] Sajid MS, Betal D, Akhter N, et al. Prevention of postoperative seroma-related morbidity by quilting of latissimus dorsiflap donor site: a systematic review. Clin Breast Cancer 2011;11(6):357-63.

[21] Barry M, Kell MR. Radiotherapy and breast reconstruction: a meta-analysis. Breast Cancer Res Treat 2011;127(1):15-22.

[22] Brandberg Y, Arver B, Johansson $H$, et al. Less correspondence between expectations before and cosmetic results after risk - reducing mastectomy in women who are mutation carriers: a prospective study. Eur J Surg Oncol 2012;38(1):38-43.

[23] Gilmour A, Mackay IR, Young D, et al. The use of realtime digital video in the assessment of post-operative 
outcomes of breast reconstruction. J Plast Reconstr Aesthet Surg 2014;67(10):1357-63.

[24] Asplund 0, Nilsson B. Interobserver variation and cosmetic result of submuscular breast reconstruction. Scand J Plast Reconstr Surg 1984;18(2):215-20.

[25] Brandberg Y, Malm M, Blomqvist L. A prospective and randomized study, "SVEA," comparing effects of three methods for delayed breast reconstruction on quality of life, patient-defined problem areas of life and cosmetic result. Plast Reconstr Surg 2000;105(1):66-74; discussion 75 .

[26] Cardoso MJ, Cardoso J, Santos AC, et al. Interobserver agreement and consensus over the esthetic evaluation of conservative treatment for breast cancer. Breast 2006;15(1):52-7. 